\section{Emotional geriatric care: a health worker development framework for advancing health care for the elderly}

\author{
Nestor Asiamah, ${ }^{1}$ Samuel A. Azinga ${ }^{2}$ \\ ${ }^{1}$ Africa Center for Epidemiology, Accra; \\ ${ }^{2}$ Kwame Nkrumah University of Science \\ and Technology, Kumasi Ghana
}

\begin{abstract}
The trend of population ageing is a critical global condition and is expected to impose dire socio-economic ramifications on populations in the near future. Its contribution to the global burden of disease is increasing, and the implications of this situation for the future are awe-inspiring. This paper draws from the researchers' experience and relevant literature to recommend a framework that would guide the development and institutionalization of a specialized system of geriatric care, hereby called emotional geriatric care. The application of this framework by governments and health care organizations is expected to constantly improve care for the elderly as a way of gearing health care administration in response to ageing.
\end{abstract}

\section{Introduction}

The world is on the brink of a demographic turnaround: children have always outnumbered their elders, but in the next one decade, the number of people aged 65 or more will outnumber children under age $5 .{ }^{1}$ The number of people aged 65 or older is projected to grow from an estimated 524 million in 2010 to nearly 1.5 billion in $2050 .{ }^{1}$ Owing to falling fertility rates and a significant increase in life expectancy, ${ }^{1,2}$ population ageing is expected to continue on a global stage. The world faces an unprecedented situation of ageing indeed!

Ageing is associated with deteriorating health - people become less healthy and active as they grow older. ${ }^{3,4}$ Hence, the situation of ageing in the world implies that many more people will soon have poor health. The number of people with conditions like cardiovascular disease and dementia, particularly Alzheimer's disease, will increase significantly. People with the foregoing diseases need the care and assistance of others to be able to cope with basic daily activities and situations.
This being the case, a serious level of socio-economic burden will be created by older people with these diseases. There is thus no doubt that the world will soon be populated by people with relatively poor health and physical ability.

The world apparently needs to act without delay to control and minimize the socioeconomic burden of population ageing. Special care within health organizations is highly recommended in the literature ${ }^{1,5,6}$ as a way of minimizing health problems and disabilities associated with ageing. Special care in this context is ultimately about caring for older people in an attempt to cure or treat their diseases and most importantly prevent them from being diseased.

In this paper, an attempt is made to recommend a health care policy framework, hereby called emotional geriatric care that may guide governments and health organizations in developing an effective model for caring for older people or for administering the said special care model. The framework recommended in this paper is also expected to guide academic debate towards developing a resilient model for geriatric care. This study is basically addressed to governments, health care organizations, and researchers, especially those in regions like Asia where ageing poses an enigmatic issue.

\section{How special are the health care needs of older people?}

Cardiovascular diseases are some of the commonest conditions found in older populations. ${ }^{1,7}$ Older people who suffer from these diseases, especially stroke and Alzheimer's disease, are often inactive and less self-supporting. As a consequence, they need the care and assistance of relatives to be able to cope with basic daily activities such as attending to nature's call, bathing, and visiting a physician. At extremely old age, people become less active, develop scaly skins, and lose the ability to keep themselves clean and physically appealing. Sadly, this situation does not support their access to health care because they need to be assisted by others or/and carried in a vehicle to visit the physician. This problem is compounded when the elderly person loses the ability to speak and hear. It is consequently necessary for health workers to be careful, patient, empathetic, and tolerant in providing care to the elderly, and they should be able to understand and address the special emotional needs of geriatric patients. From this standpoint, emotional geriatric care is needed in health care organizations.
Correspondence: Nestor Asiamah, Africa Center for Epidemiology, Accra, Ghana. E-mail: nestor.asiamah@yahoo.com

Key words: Emotional intelligence; ageing; geriatric care.

Conflict of interest: the authors declare no conflict of interest.

Received for publication: 11 April 2017. Accepted for publication: 15 June 2017.

This work is licensed under a Creative Commons Attribution-NonCommercial 4.0 International License (CC BY-NC 4.0).

(C) Copyright N. Asiamah and S.A. Azinga, 2017 Licensee PAGEPress, Italy

Geriatric Care 2017; 3:6741

doi:10.4081/gc.2017.6741

\section{What emotional geriatric care is?}

Older populations require sustainable access to health care, without which morbidity and age-related disability within them may pose serious social and economic ramifications. The effectiveness of national health care systems would as a result determine the quality of life of older people. Older people, as established earlier, have special health care needs. Hence to ensure effectiveness in health care for them, organizations and governments must be able to tailor a special system of geriatric care whose functionality is not conflicted by the traditional health care system. More importantly, the human resource component of this system must be able to perform three tasks: withstanding the deterring attributes of old age; understanding the emotional and physical conditions of elderly people; and appropriately responding to these conditions in health care.

The ability of health care professionals to perform these three tasks would make a major impact on the quality of the relationship they have with geriatric patients. Needless to say, health care is largely about relating with patients and ensuring that the personnel-patient experience translates to effective care. Hence, the fulfillment of these tasks by the health worker is pivotal to result-oriented care in which the caregiver relates well with the patient. It is argued in this study that the ability to relate well with older people in health care largely includes emotional intelligence (EI). In this study, therefore, emotional geriatric care is a term used to describe health care for older people that is largely influenced by the application of EI. To better explain this term, an attempt 
is made to explain what EI is in the next section.

\section{What is emotional intelligence?}

Emotional intelligence is the ability to accurately perceive emotions, to access and generate emotions so as to assist thought, to understand emotions and emotional knowledge, and to reflectively regulate emotions so as to promote emotional and intellectual growth. ${ }^{8,9}$ This definition is similar to Salovey and Mayer's 9 conceptualization of EI, which recognizes EI as an embodiment of four skills: i) the accurate perception, appraisal, and expression of people's emotions; ii) generating feelings on demand when they can facilitate an understanding of self or others; iii) understanding emotions and the knowledge that can be derived from them; and iv) regulation and control of emotions to promote emotional and intellectual growth.

The above conceptualization of EI is considered the root of modern EI definitions and models. It suggests that EI is the ability to appraise emotions of self and those of the elderly people, understand these emotions, and relish this understanding to address health care needs. It includes the ability to align one's emotions with those of geriatric patients as a basis of identifying and positively responding to their health care needs. The ability to respond to health care needs in this context is basically about using an approach that yields effective care.

The concept of EI is noteworthy in the literature partly because several studies have confirmed its ability to predict key performance indicators, namely health care performance, ${ }^{10-12}$ leadership in health organizations, ${ }^{13,14}$ and health care quality. ${ }^{15,16}$ This body of studies has expanded enormously to the extent that many researchers $8,13,16$ have been encouraged to acknowledge the need for institutions to equip their workers with EI. The euphoria has also impelled some researchers $8,10,11,16,17$ to suggest the need for health organizations to adopt and implement career development programs that would more effectively grow the EI of their employees over time. Asiamah ${ }^{8}$ further empirically justified the importance of specializing training and development programs in health care institutions for enhancing the EI of health care personnel, whereas originators of the concept of EI such as Bar$\mathrm{On}^{18}$ have attempted to develop and recommend specialized EI training models. Given this progress made in the school of EI, it is apparent EI is a necessary health care skill.
The concept of emotional geriatric care can as a result become a framework for promoting the quality and performance of health care for older people if properly institutionalized.

\section{Institutionalizing emotional geriatric care}

The body of studies that has confirmed the positive association between EI and health care performance and quality is constantly growing. Though EI research is still lacking in many jurisdictions and little of the foregoing empirical evidence exists on some countries, ${ }^{11,12}$ the majority of studies have indicated that the application of EI in health care contributes to health care quality and performance enhancement. ${ }^{11}$ Consequently, health care for the elderly can be enhanced by increasing the level of health workers' EI. It is argued in this study that EI will make a stronger impact on geriatric care because caring for older people is largely about meeting stronger and more sensitive emotional needs. As population ageing becomes more of a global issue, governments and health care administrators would want health workers to be emotionally intelligent.

Fortunately, every human being is born with EI that provides a basis for intellectual capacity building and interpersonal relationship development. ${ }^{13}$ The level of EI can also be increased continuously to augment its impact on how the individual relates well with people. So, every health professional has a level of EI that can be developed and increased. Based on Asiamah, ${ }^{12}$ increasing the level of EI requires the application of specialized methods in an organizational Career Development Program (CDP). We are of the opinion that the best way to administer these methods to advance geriatric care is to institutionalize emotional geriatric care as explained in the following paragraphs.

\section{Creating a more specialized care system for older people}

Creating a more specialized health care system for older people is a contingency step driven by the alarming situation of ageing. While many health care organizations already have a specialized care environment for older people in the form of a geriatric medical department, any existing or potential geriatric care department needs to be rejuvenated to respond to ageing by: i) harnessing a higher level of government support for geriatric care and upholding the need for health care systems to be modern- ized to meet the burden of ageing; ii) improving geriatric care infrastructure and resources within hospitals to avoid competition between the geriatric medical department and traditional departments; and iii) influencing health care professionals through CDPs to apply skills (e.g., EI) pertinent to geriatric care.

\section{Incorporating emotional intelligence in geriatric care policy}

Health care within nations and organizations is based on a policy framework, often referred to as healthcare policy. This being the case, reviewing the current health care policy to support continuous development of the EI of health workers using CDPs is a necessary foundation for institutionalizing geriatric care. More often than not, national health care facilities are owned and regulated by government. Therefore, health care policies in these facilities are developed or are at least sanctioned by government. Getting the continuous EI development program entrenched in health care policy is therefore the responsibility of two key stakeholders, namely governments and health care organizations.

Emotional intelligence can be incorporated in health care policy in three ways: i) modifying the existing health care administration policy to adopt a culture of EI-driven care for older people; ii) reviewing existing CDPs in the organization to enable the use of training and other employee development programs to increase health workers' EI level; and iii) developing and nurturing an EI maturity improvement program that is aimed at enhancing the level of health personnel's EI over time.

\section{Using Career Development Program to enhance the emotional intelligence of geriatric caregivers}

Emotional intelligence is a malleable skill ${ }^{13,14}$ and can as a result be developed to increase its impact on everyday behavior. Governments and health care organizations, therefore, have the opportunity to improve EI and its impact on geriatric care. So, the institutionalization of emotional geriatric care is incomplete without using CDPs in health care organizations to increase the level of health workers' EI. In this study, the use of CDPs to enhance the EI of geriatric caregivers is about using three specialized methods, which are on-the-job training, formal education, and tenure prolongation. The applicability of these methods to enhance EI and its impact on geriatric care is supported by several studies. , $^{8,111,15-18}$

Goleman's ${ }^{13}$ theory asserts that EI is malleable and can be developed over time. 
From the point of view of this theory, an individual who is able to exercise his nature-given EI through some life experiences can increase his level of EI. The theory thus implies that EI can increase with job tenure if health workers significantly encounter job conditions that enable them to exercise their EI. This argument is supported by a team of researchers, ${ }^{19}$ who confirmed that EI increases with job tenure among health workers.

On the contrary, many studies ${ }^{12,13}$ have also failed to confirm a significant relationship between job tenure and EI in populations of health workers. Asiamah, ${ }^{8}$ nonetheless, found in his study that job tenure is less likely to positively influence EI if CDPs for prolonging job tenure in a health care organization are not specialized or designed for EI enhancement. The import being that identifiable studies that have failed to confirm the relationship between EI and job tenure drew their data from institutions in which such non-specialized programs were applied. Hence, this specialization program is necessary for realizing a positive association between job tenure and EI, which is represented by $\mathrm{H}_{\mathrm{a} 1}$ in Figure 1 . The specialization of CDPs and tenure prolongation programs is thus necessary in the institutionalization of emotional geriatric care. A question worth answering is How can tenure prolongation be specialized for EI enhancement?.

Several work conditions influence the tenure of employees on the job. A satisfactory pay, fringe benefits, recognition, a favorable work condition, and an opportunity to develop a career on the job are some of these conditions. But to specialize an organization's tenure prolongation program so as to constantly improve EI and its expected impact on geriatric care as represented by $\mathrm{H}_{\mathrm{a} 2}$ in Figure 1, these conditions must be coupled with two traditional practices for building competencies, namely on-the-job training and formal education. To explain, health workers must be made to regularly participate in on-the-job training programs specially designed to improve EI. On-thejob training can be specialized to enhance the level of EI and its positive effect on geriatric care by using EI-focused training models and special trainers. ${ }^{16,18}$

Increasing the formal education of health care personnel is another potentially effective way to improve skills such as $\mathrm{EI}, 8,10,16,19$ though this approach is also more likely to positively influence EI if specialized. A good way to specialize formal edu- cation is incorporating special modules in the curriculum of health worker training in an attempt to introduce health care personnel to what they can do to build their naturegiven EI over time. These modules may include practical work in which trainees are made to engage in an activity for exercising and building their EI.

Prior to taking the above steps, governments must include EI-oriented modules in the curriculum of health worker training programs, making it possible for EI improvement to develop its roots in trainees' educational programs. The likelihood of formal education causing an increase in health workers' EI level, as represented by $\mathrm{H}_{a 3}$, is increased when this and the above steps are taken by government. Given the much empirically confirmed positive relationship between EI and health care quality and performance, there is no doubt that EI can cause an improvement (which is represented by $\mathrm{H}_{\mathrm{a} 4}$ in Figure 1) in geriatric care if on-the-job training, tenure prolongation, and formal education are specialized to enhance EI. The enhancement of EI in health care organizations, however, must be based on a maturity assessment culture. With respect to Figure 1, the paths $\mathrm{H}_{\mathrm{a}}$, $\mathrm{H}_{\mathrm{a} 6}$, and $\mathrm{H}_{\mathrm{a} 7}$ suggest that on-the-job train-

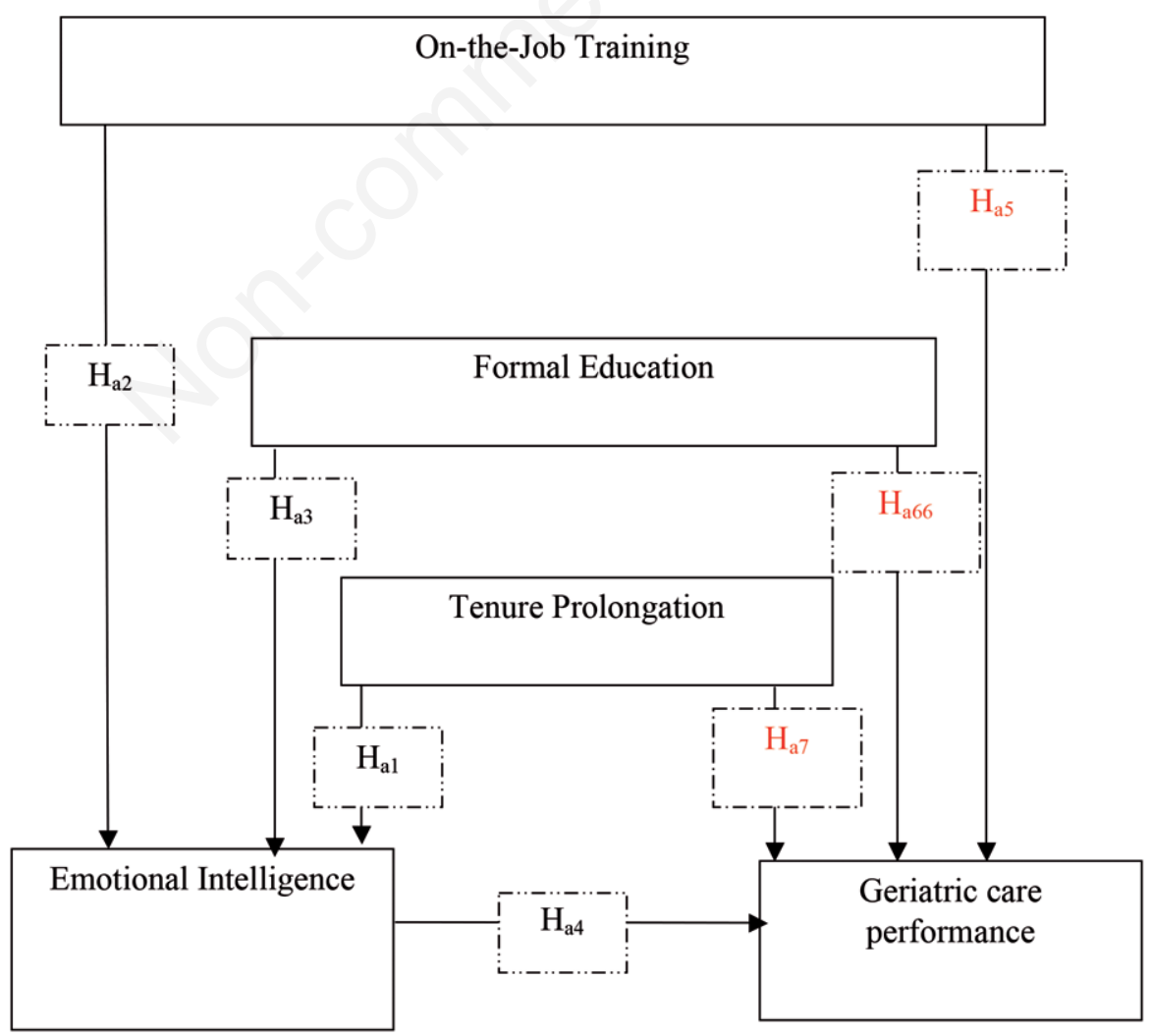

Figure 1. A model for improving emotional intelligence (EI) and geriatric care through a Career Development Program (CDP). 
ing, formal education, and tenure prolongation can also make a direct effect on geriatric care performance.

\section{Adopting an emotional intelligence maturity assessment}

Using the above specialization program to advance the EI of health workers is of little significance without running an EI enhancement monitoring system, hereby called 'EI maturity assessment'. This system is about taken three key steps in a health care organization as part of the institutionalization of emotional geriatric care: i) taking the specialized steps mentioned earlier continuously to augment EI and the ability of workers to apply it in health care for the elderly; ii) establishing a maturity assessment scale on which the EI of all geriatric caregivers is measured and evaluated each year. Authorities can develop a scale ranging from lowest EI level to highest EI level that are respectively assigned the codes 1 and 10. The year in which the institutionalization of emotional geriatric care commences is the base or reference year. With respect to the maturity assessment program, personnel's EI level for the reference year should take any value between 1 and 10. A similar scale can be developed for assessing improvement in geriatric care. It may not be appropriate to use traditional validated scales in assessing EI and geriatric care in this process because their range of values, which is far smaller than the scale recommended above, may be too small to support the continuous EI and geriatric care improvement goal for a significant period; iii) applying the recommended CDPs in the organization to ensure that the EI of health workers and its impact on geriatric care increase year after year along the range of values making up the maturity scale acknowledged supra. For instance, if the EI level of personnel in the base year is 1.5 , the specialized steps taken in the base year should increase this value of EI to say 2. If a fall in personnel's EI is encountered instead of an increase, the specialized steps must be questioned, reviewed, and improved. Steps can be improved by making on-the-job training more frequent, using more focused EI training models and trainers, and increasing the level of EI-focused orientation given to trainee health workers; iv) carrying out an annual evaluation exercise to find out the EI level of the workforce and its impact on geriatric care performance. This effort would enable authorities to know whether or not personnel's EI and its impact on geriatric care have increased vis-à-vis their respective levels for the previous year. The same maturity scale ought to be used in the organization until the EI of the workforce reaches its maximum value, 10 . The maximum value of the scale would have to be increased to a tangible value, say 20 , to warrant continuity of the assessment program.

\section{Conclusions}

Older people have special health care needs that can be better met when their caregivers are empowered to apply emotional intelligence. From this perspective, emotional geriatric care would have to be institutionalized in health care organizations by recognizing EI as a skill relevant to health care for the elderly; creating a more specialized care system for older people; incorporating EI in geriatric care policy; using CDPs to enhance the EI of geriatric caregivers; and adopting an EI maturity assessment culture in accordance with Figure 2. This figure depicts five systematic phases represented by five squares linked by four arrows (i.e., A-B-C-D). These phases represent steps that can be taken to introduce and permanently practice emotional geriatric care.

\section{Limitations and suggestions for future research}

We admit that research on EI in terms of how it impacts health care, especially geriatric care, is scant in many jurisdictions. The applicability of our framework is therefore subject to the empirical confirmation of EI as a skill relevant to geriatric care in specific areas and health care settings. In addition, some studies ${ }^{8}$ have justified that onthe-job training, formal education, and

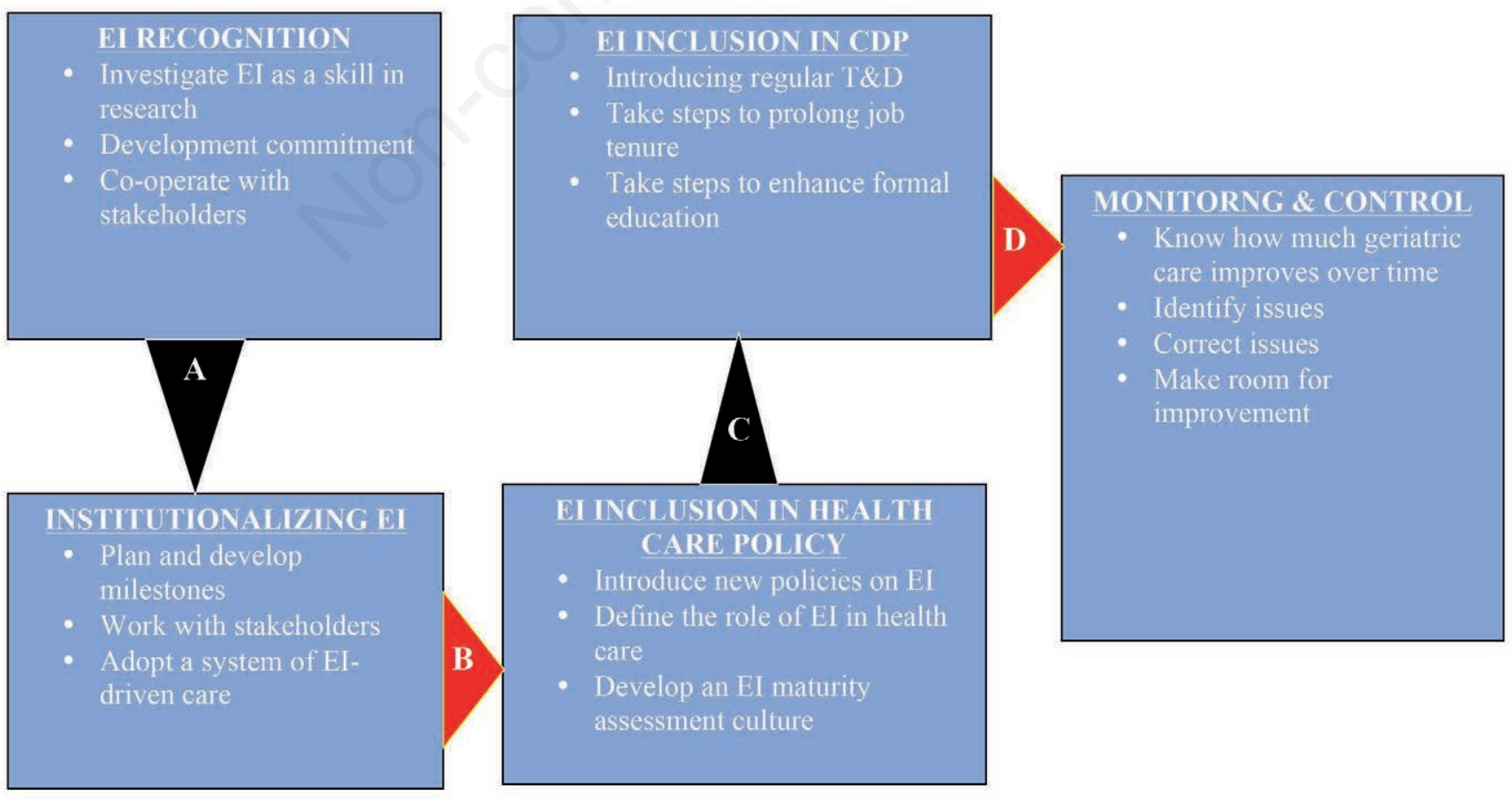

Figure 2. A framework for institutionalizing emotional geriatric care. 
tenure prolongation need to be specialized for EI improvement in order to significantly cause an increase in the level of health workers' EI. Unfortunately, research has not sufficiently shown that these programs consistently cause an increase in the level of EI of health workers when specialized. Our assumption that they make a positive effect on the EI of health workers is consequently not sufficiently backed in the literature. Moreover, the scale suggested in this paper for assessing EI and geriatric care maturity is different from traditional validated scales. Hence, its credibility in the context of the maturity assessment process is not guaranteed and would have to be investigated in future research.

\section{References}

1. Global Health and Ageing. U.S. Department of Health and Human Services; 2011. Available from: http:// www.who.int/ageing/publications/global_health.pdf Accessed: March 12, 2017.

2. Tchoe B, Nam S. Aging risk and health care expenditure in Korea. Int J Environ Res Public Health 2010;7:3235-54.

3. Teo P. Ageing in Singapore. J CrossCultural Gerontol 1996;11:269-86.

4. Wong CY, Lee HC. Healthcare in
Singapore: challenges and management. Int Med Commun 2008;51:343-6.

5. Oliver D, Foot C, Humphries R. Making our health and care systems fit for an ageing population. London: The King's Fund; 2014. pp 1-82.

6. Phua KH. Ageing: socio-economic implications for health care in Singapore. Ann Acad Med 1987;16:15-23.

7. Digby R, Lee S, Williams A. Nurse empathy and the care of people with dementia. Austr J Adv Nursing 2016;34: 52-9.

8. Asiamah N. Enhancing nurses' emotional intelligence: Are tenure prolongation, education and in-service training applicable methods even when not specialized? Cogent Business Manage 2017;4: $1-16$.

9. Salovey P, Mayer JD. Emotional intelligence. Imagin Cognit Personal 1989;9: 185-211.

10. Asiamah N. The Nexus between health workers' emotional intelligence and job performance: controlling for gender, tenure, education and in-service training. J Global Respons 2017;8:1-24.

11. Shannon C, Madeline J, Lee W, et al. An exploration of emotional intelligence and job performance among nurses in rural Texas. Am Int J Contemp Res 2013;3:1-6.

12. Farooq MU, ur Rehman K. Emotional intelligence and organizational productivity: a conceptual study. World Appl Sci J 2011;15:821-5.

13. Goleman D. Emotional Intelligence. New York, NY: Bantam Books, Inc.; 1995.

14. Goleman D. Working with emotional intelligence. New York, NY: Bantam Books, Inc.; 1998.

15. Birks YF, Watt IS. Emotional intelligence and patient-centered care. J Roy Soc Med 2007;100:369-74.

16. Freshman B, Rubino L. Emotional intelligence: a core competency for health care administrators. Health Care Manag 2002;20:1-9.

17. Juhásová I, Ilievová L, Baumgartner F, et al. Emotional intelligence of nursing students and its role in interactions with geriatric patients. Profese on-line 2013;6:12-6.

18. Bar-On R. Development of the Bar-On EQ-i: a measure of emotional and social intelligence. Chicago, IL: 105th Annual Convention of the American Psychological Association; 1997.

19. Asiamah N, Mensah HK, Oteng-Abayie EF. Health workers' emotional intelligence development: an examination of the potential roles of tenure, education and training. Int J Res Nursing 2016;7: 24-34. 